\section{MS19-05 Templation effects and novel ZIF structures by solid state synthesis}

Ivana Brekalo ${ }^{1}$, Joseph R. Ramirez ${ }^{1}$, Christopher M. Kane ${ }^{1}$, K. Travis Holman ${ }^{1}$

1. Department of Chemistry, Georgetown University, Washington, D.C. 20057, USA

email: ib308@georgetown.edu

In the last decade zeolitic imidazolate frameworks (ZIFs), a subclass of metaloorganic frameworks (MOFs), have been rising in popularity due to their diverse topologies, good thermal and chemical stability, and large pore volumes, which could enable their use for gas-storage, separation of gases, and catalysis. ${ }^{1}$ However, traditional solution synthesis methods are very energy demanding, they use large quantities of organic solvents and rather expensive inorganic nitrates as starting material, and, above all, are often irreproducible and hard to control, giving mixtures of different ZIF frameworks in often low yields. Mechanochemical alternatives have been proposed, including neat grinding ${ }^{2}$, ion and liquid assisted grinding (ILAG) ${ }^{3}$, and most recently, accelerated aging $^{4}$.

We explored several different methods for solid state ZIF synthesis, including neat and liquid-assisted grinding, slurrying, as well as a modified accelerated aging procedure, giving rise to novel ZIF structures. We also show that we can direct the outcome of ZIF syntheses to frameworks containing a specific structural motif by employing an organic template using several different synthetic methods.

[1] Park, K.S.; Ni, Z.; Côté, A. P.; Choi, J. Y.; Huang, R.; Uribe-Romo, F. J.; Chae, H.H.; O'Keeffe, M.; Yaghi, O. M. Proc. Natl. Acad. Sci. U. S. A. 2006, 103, 10186-10191.

[2] Tanaka, S.; Kida, K.; Nagaoka, T.; Otaa, T.; Miyake, Y.; Chem. Commun., 2013, 49, 7884-7886.

[3] Beldon, P. J.; Fabian, L.; Stein, R. S.; Thirumurugan, A.; Cheetham, A. K.; Friščić, T. Angew. Chem. Int. Ed. 2010, 46, 9640-9643

[4] Cliffe, M. J.; Mottillo, C.; Stein, R. S.; Bučar, D.-K.; Friščić, T. Chem. Sci., 2012, 3, 2495.

Keywords: zeolitic imidazolate frameworks, solid-state synthesis, templation

\section{MS20. High pressure solid}

state chemistry

Chairs: Natalia Dubrovinskaia, Wilson Crichton

MS20-01 High-pressure high-temperature synthesis of new covalent metals

Ulrich Schwarz ${ }^{1}$

1. Max Planck Institute for Chemical Physics of Solids, Dresden, Germany

email: Ulrich.Schwarz@cpfs.mpg.de

In the last two decades, the advent of modern in-situ high-pressure $\mathrm{x}$-ray diffraction techniques has markedly developed our knowledge of exotic structure patterns. The alteration of atomic configurations opens perspectives to substantially manipulate the electronic properties of solids. The increased number of neighbors in the atomic coordination sphere may give rise to an enlarged band dispersion and evenually metallization. Particularly in covalent framework assemblies, the combination of directed bonding with a significant density of states at the Fermi level creates the promising situation of covalent metals. This unique blend of properties has proven to be beneficial for phonon-mediated superconductivity.

This way, high-pressure studies have supplemented the map of high-temperature superconductors as much as they stimulated profound studies of the underlying principles. A straightforward method to access the useful effects of compression at ambient pressure is provided by synthesizing metastable compounds at extreme conditions. Recent preparation results and physical propeties of selected binary compounds comprising covalent framework patterns will be discussed.

\section{References}

A. Wosylus, Yu. Prots, U. Burkhardt, W. Schnelle, U. Schwarz, STAM 8, 383 (2007). K. Meier, R. Cardoso-Gil, W. Schnelle, H. Rosner, U. Burkhardt, U. Schwarz, ZAAC 636, 1466 (2010). K. Meier, L. Vasylechko, R. Cardoso-Gil, U. Burkhardt, W. Schnelle, M. Schmidt, Yu. Grin, U. Schwarz, ZAAC 636, 1695 (2010). A. Wosylus, K. Meier, Yu. Prots, W. Schnelle, H. Rosner, U. Schwarz, Yu. Grin, Angew. Chemie Int. Ed. 49, 9002 (2010). W. Schnelle, A. Ormeci, A. Wosylus, K. Meier, Yu. Grin, U. Schwarz, Inorg. Chem. 51, 5509 (2012). K. Meier, A. Wosylus, R. Cardoso-Gil, U. Burkhardt, C. Curfs, M. Hanfland, Yu. Grin, U. Schwarz, ZAAC 638, 1446 (2012). U. Schwarz, A. Wosylus, H. 
Rosner, W. Schnelle, A. Ormeci, K. Meier, A. Baranov, M. Nicklas, S. Leipe, C. J. Müller, Yu. Grin, JACS 134, 13558 (2012). U. Schwarz, S. Tencé, O. Janson, C. Koz, C. Krellner, U. Burkhardt, H. Rosner, F. Steglich, Yu. Grin, Angew. Chemie Int. Ed. 52, 9853 (2013).

Keywords: hifh-pressure synthesis, binary compounds, metastable phases

\section{MS20-O2 Crystal chemistry of postperovskite-type $A M X_{3}$ compounds \\ Yuichi Shirako ${ }^{1,2,3}$, Youguo Shi ${ }^{4,5}$, Jianshi Zhou ${ }^{2}$, Kazunari Yamaura $^{4}$, Masashi Hasegawa ${ }^{3}$, Masaki Akaogi ${ }^{1}$}

1. Department of Chemistry, Gakushuin University, Japan 2. Texas Materials Institute, Cockrell School of Engineering, University of Texas at Austin, USA

3. Department of Crystalline Materials Science, Nagoya University, Japan

4. Superconducting Properties Unit, National Institute for Materials Science, Japan

5. Institute of Physics, Chinese Academy of Science, P. R. China

email: shirako@numse.nagoya-u.ac.jp

Since the discovery of postperovskite type $\mathrm{MgSiO}_{3}$, $A M X_{3}$ compounds with the postperovskite structure have attracted special attention for a recent decade in not only mineral physics but also condensed matter physics and materials science due to its alternative layered structure (ref. 1-6). Crystal chemistry of the postperovskite compounds has been quickly developed to seek for new postperovskite materials that are potentially useful in a variety of technology and science, regarding such as superconductivity and correlated electronics. It has been argued that ionic size and covalency are significant in stabilizing the postperovskite structure (ref. 7-10). Regarding ionic size, one can easily understand its effects on the tolerance factor and octahedral-site rotation of perovskite which may affect stability of the perovskite structure, leading to the perovskite to the postperovskite transition. However, in contrast, effects of bond covalency seem to be studied little over the perovskite-postperovskite transition. Therefore, no postperovskite compounds have been designed from a view point of covalent character except strong covalency in contrast to that of ionic size.

In this presentation, crystal chemistry of postperovskite materials in terms of nature of covalent bonds is discussed. These results indicate that the electron count of outermost shell of the cation which makes stronger covalent bond with anion than the other cation or total number of valence electrons of cluster ions is important as well as the electronegativity. It seems to be qualitatively similar with what was developed by Burdett et al for the other, some solid-state structures (ref. 11, 12). In addition, we will also report on a new material which was predicted based on these considerations.

[1] Murakami et al., Science, 304, 855 (2004).

[2] Yamaura et al., J. Am. Chem. Soc., 131, 2722 (2009).

[3] Cheng et al., Phys. Rev. B, 83, 064401 (2011).

[4] Shirako et al., Phys. Rev. B, 83, 174411 (2011).

[5] Ohgushi et al., Phys. Rev. Lett., 110, 217212 (2013).

[6] Bernal et al., Inorg. Chem., 53, 12205 (2014).

[7] Martin et al., Am. Miner., 92, 1912 (2007).

[8] Ohgushi et al., Phys. Chem. Miner., 35, 189 (2008). 